\title{
Emotional context at learning systematically biases memory for facial information
}

\author{
Donna J. Bridge, Joan Y. Chiao, and Ken A. Paller \\ Northwestern University, Evanston, Illinois
}

\begin{abstract}
Emotion influences memory in many ways. For example, when a mood-dependent processing shift is operative, happy moods promote global processing and sad moods direct attention to local features of complex visual stimuli. We hypothesized that an emotional context associated with to-be-learned facial stimuli could preferentially promote global or local processing. At learning, faces with neutral expressions were paired with a narrative providing either a happy or a sad context. At test, faces were presented in an upright or inverted orientation, emphasizing configural or analytical processing, respectively. A recognition advantage was found for upright faces learned in happy contexts relative to those in sad contexts, whereas recognition was better for inverted faces learned in sad contexts than for those in happy contexts. We thus infer that a positive emotional context prompted more effective storage of holistic, configural, or global facial information, whereas a negative emotional context prompted relatively more effective storage of local or feature-based facial information.
\end{abstract}

Analysis of both local and global information is essential to efficiently process and remember many types of stimuli. A variety of factors can influence the degree to which a perceiver's focus is directed to local or global information conveyed by stimuli such as hierarchical letters (e.g., Navon, 1977) and human faces (e.g., Tanaka \& Farah, 1993). In particular, some types of positive affect can broaden the scope of attentional selection relative to neutral and negative affect (Fredrickson \& Branigan, 2005; Gable \& Harmon-Jones, 2008; Rowe, Hirsh, \& Anderson, 2007). For instance, when asked to judge the similarity of hierarchical shapes such as a large triangle constructed of little squares, happy participants tended to focus on global form as a basis for their relational comparisons, whereas sad participants relied on the local components as a means of comparison (Gasper \& Clore, 2002).

A mood-induced holistic bias may be particularly beneficial for face perception and for face memory, given that expertise with faces is thought to depend on configural processing (Maurer, Le Grand, \& Mondloch, 2002). Drawing from evidence suggesting that positive mood broadens the attentional scope of visual processing, Johnson and Fredrickson (2005) manipulated the mood of participants and tested recognition memory for same- and other-race faces with neutral expressions. The other-race effect, which is marked by a decrement in memory performance for other- relative to same-race faces (Golby, Gabrieli, Chiao, \& Eberhardt, 2001), was attenuated for the participants put into happy moods relative to those put into negative moods. The mood manipulation had no effect on memory for same-race faces; rather, happy moods led to an enhancement of memory only for other-race faces. Ac- cordingly, a holistic processing preference may have been engaged by default when viewing same-race faces but not other-race faces. Moreover, a default holistic processing mode may be associated with the individuation most consistently applied when people view same-race faces.

Another way in which emotion can influence memory formation, possibly distinguishable from attentional modulation as a function of the mood of the observer, emerges when to-be-remembered stimuli have inherent emotional valence. Emotional stimuli are known to influence memory processes at various levels, beginning at encoding and extending to consolidation and retrieval stages (Payne, Stickgold, Swanberg, \& Kensinger, 2008; Phelps, 2004). Several studies have demonstrated boosts in recollection (Ochsner, 2000) and in memory for details of negative visual scenes and objects (Canli, Zhao, Brewer, Gabrieli, \& Cahill, 2000; Kensinger, Garoff-Eaton, \& Schacter, 2007a, 2007b). However, these memory enhancements do not necessarily come without a cost. A memory trade-off, for example, was found with emotionally negative objects projected onto neutral backgrounds (Kensinger et al., 2007b). Recognition of central objects was superior for negative relative to neutral objects, whereas recognition of backgrounds was superior for those associated with neutral objects relative to those associated with negative objects. Thus, recognition memory can be enhanced for one component of a visual scene when it is negative, but memories for neutral components of the same scene are simultaneously weakened.

Recognition memory for faces can also be influenced by inherent emotional valence, especially as conveyed through facial expressions. Happy faces are recognized

D. J. Bridge, donnajb@u.northwestern.edu 
better than angry, sad, or neutral faces (D'Argembeau, van der Linden, Comblain, \& Etienne, 2003; Marian \& Shimamura, 2004; Ridout, Astell, Reid, Glen, \& O'Carroll, 2003). An advantage for happy faces can also be found when emotional expressions are only processed subliminally (Sweeny, Grabowecky, Suzuki, \& Paller, 2009) or when attention is not oriented on the facial expression during encoding (D'Argembeau \& van der Linden, 2007). This memory advantage for happy faces may be due to a boost in holistic processing that is similar to the aforementioned results obtained by Johnson and Fredrickson (2005). In accordance with these ideas about mood and processing strategy, clinically depressed patients do not show a memory advantage for happy faces; rather, they remember sad faces better than happy faces (Ridout et al., 2003). This memory effect may be attributed to the overall sad mood of the patients, which could cause them to emphasize an abnormal processing strategy when viewing faces. In addition, Shimamura, Ross, and Bennett (2006) observed a source-memory advantage for happy facial expressions; participants remembered the category of emotional expression more accurately when viewing neutral test faces that had displayed happy facial expressions than when viewing those that had other emotional expressions during the study phase.

Memory experiments with faces displaying emotional expressions, however, may simultaneously engage two quite different mechanisms biasing memory performance. Both the stimulus characteristics of a happy face and the positive emotional valence that the face provides, for example, can potentially influence memory storage. Facial expressions change the appearance of a face through configural alterations, featural alterations, or both (e.g., curled sides of the mouth moved upward and eyes squinted to smile). Some aspects of a facial expression might make a face easier to remember, independent of any perceived emotion. The influence of emotional valence per se in the memory advantage for happy faces is thus difficult to determine. Moreover, some expressions may draw the observer's attention to particular features of the face that are rich in emotional information. For instance, fear is conveyed with wide-open eyes, and this feature must be visually encoded to correctly identify the emotional valence of the face (Adolphs et al., 2005). When attention is thereby directed to one facial feature, a memory trade-off may ensue such that memory suffers for other facial features, or for the facial configuration as a whole.

In order to disentangle effects of emotional valence and facial expressions on recognition memory, special experimental procedures are needed. Fortunately, to-beremembered facial images can be held constant while manipulating emotion, given that emotions can be conveyed in other ways. Indeed, the perceived valence of a face displaying an ambiguous facial expression can be manipulated with a contextual description that accompanies the face (Kim et al., 2004). Similarly, the interpretation of a facial expression conveyed by a person in a visual scene can be modulated by the context of the scene (Barrett, Lindquist, \& Gendron, 2007).
What impact would emotional context have on facial memory in such circumstances? In keeping with Fredrickson and Branigan (2005), a positive context would be expected to broaden the scope of attention, whereas a negative context might lead participants to direct their attention to details. Mapping this onto faces, positive contextual cues would produce a configural processing bias, whereas negative contextual cues would elicit a local or part-based processing bias. Given the notion that face inversion disrupts configural processing but preserves local processing of facial components (e.g., Diamond \& Carey, 1986; Maurer et al., 2002; Tanaka \& Farah, 1993; Valentine, 1988), and if these assumptions about global and local factors hold, a memory advantage for faces from a positive context would be expected when memory is tested with upright facial images, but a memory advantage for faces from a negative context would be expected when memory is tested with inverted facial images.

We thus developed an experimental design to determine whether emotional context, rather than emotional expressions per se, would influence the processing strategy employed during encoding of neutral faces and bias subsequent recognition memory for those faces. We tested recognition memory for neutral faces learned in either a happy or sad context. For example, an observer would see a neutral face in a context provided by the sentence, "Richard was in a car accident and is now paralyzed from the waist down." The observer would imagine that the person pictured was the protagonist in the vignette. We tested memory in two ways: with upright faces, when configural processing is thought to be preferentially operative, and with inverted faces, when featural processing is thought to be preferentially operative.

\section{EXPERIMENT 1}

\section{Method}

Participants. Twenty-four Northwestern University undergraduates volunteered for the study or received course credit for their participation (13 women; mean age $=19.61$ years, $S E=0.24$ ).

Stimuli. Sixty-four neutral faces were selected from the Karolinska directed emotion face set (Lundqvist, Flykt, \& Ohman, 1998), half from each gender. The faces were cropped using an elliptical stencil to omit hair, which may distract participants from emotionally relevant facial features (Tyler \& Chen, 2006). Next, the faces were scaled to be approximately the same size with respect to the distance between the hairline and the chin and that from cheek to cheek (Figures 1A and 1B). Thirty-two emotional vignettes were created using hypothetical scenarios and names (see the Appendix). Each vignette briefly described a scenario that involved a person in a happy or a sad situation.

In a separate behavioral validation experiment, the valence associated with the vignettes was confirmed by ratings from 23 participants. Sixty-four neutral and emotional vignettes were rated on a scale of $1-9(1=$ very sad; $9=$ very happy $)$, where a response of 5 indicated neutral affect. Ratings from each vignette were normalized for statistical analysis, so that a response of 1 indicated the most extreme emotional response possible for both happy and sad vignettes, and a response of 5 indicated a neutral rating. We then averaged the ratings across participants and chose the 16 most extreme happy vignettes and the 16 most extreme sad vignettes to include in the memory study. Paired samples $t$ tests on the normalized ratings indi- 
Happy Encoding Context Block
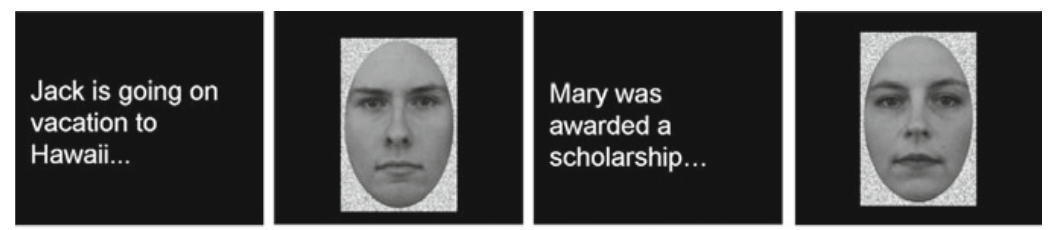

\section{Sad Encoding Context Block}

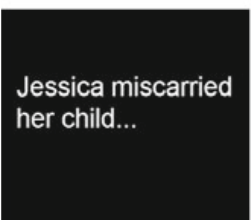

Until Response

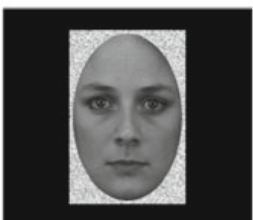

$3 \mathrm{sec}$

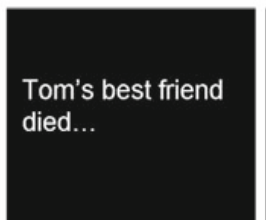

Until Response

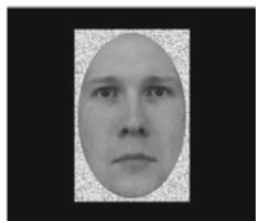

$3 \mathrm{sec}$

\section{B}

\section{New Upright Face}

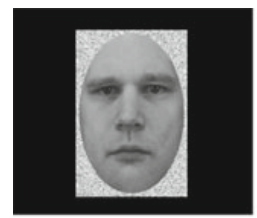

$500 \mathrm{msec}$

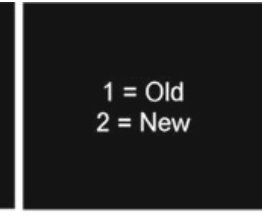

Until Response

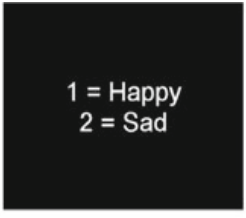

Until Response

Test Phase

\section{SEC Inverted Face}

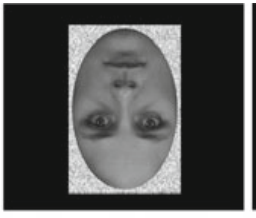

$500 \mathrm{msec}$

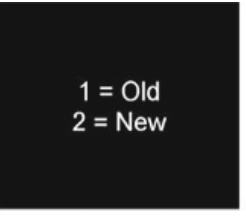

Until Response

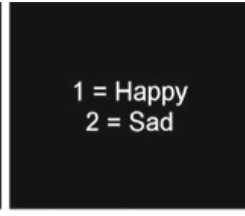

Until Response

Time

Figure 1. Examples of stimulus displays with timing parameters. (A) Study phase. Vignette-face pairings constituted a short emotional sentence followed by an upright neutral face. Individual study blocks contained eight happy or eight sad vignette-face pairings. Each vignette and each to-be-remembered face was presented only once. (B) Test phase. Half of the old and new faces were presented inverted at test. After each face presentation, the participants indicated whether the face was old or new and then identified the emotional encoding context as happy or sad. SEC, sad encoding context.

cated that the happy vignettes $(M=2.43, S E=0.14)[t(22)=17.45$, $p<.001]$ and the sad vignettes $(M=2.05, S E=0.12)[t(22)=$ $25.05, p<.001]$ were rated significantly different from neutral.

Procedure. Before the study phase, the participants were informed that they should pay careful attention to the identity of the faces that they were about to see and the emotional context associated with each face and that memory would be tested later. No additional information was given about the exact conditions of the memory test.

The study phase consisted of four blocks of vignette-face pairings. Each block included either eight happy or eight sad vignettes (Figure 1A). An unlimited amount of time was allowed for reading each vignette. Next, an upright face appeared for $3,000 \mathrm{msec}$. The participants were instructed to read each vignette carefully and to imagine that the subsequent face depicted the protagonist. Two happy-context and two sad-context blocks occurred in a randomized order.

After the study phase, the participants completed a 30-sec distractor task. In this task, the participants counted backward by increments of 3 , beginning at 975 .
The test phase followed the distractor task (Figure 1B). The participants were informed that faces would sometimes be presented in an inverted orientation during the test phase. The faces were presented in a randomized order. The test included the 16 faces learned in a happy context (happy encoding context or HEC faces), the 16 faces learned in a sad encoding context (sad encoding context or SEC faces), and 32 new faces. For each type of face, half were presented upright and the other half inverted. The participants viewed each face for $500 \mathrm{msec}$ and endorsed the face as old or new with a buttonpress response. After making a response, the participants indicated whether the face had been associated with a happy or sad context during the study phase with another buttonpress response. If they had never seen the face before or they did not remember seeing the face previously, they were instructed to guess whether the face would be classified as happy or sad.

The participants also completed a questionnaire in which they rated the emotionality of each of the vignettes. The participants' ratings were not significantly different from the ratings collected in 
the validation behavioral experiment ( $p \mathrm{~s}>.2$ for both happy and sad vignettes). Specific faces assigned to the HEC, SEC, and new conditions and to the upright and inverted conditions were counterbalanced across participants. Each specific face was thus equally likely to appear in each condition.

Data analysis. Hit rates were calculated as the percentage of correctly endorsed old faces in each of the four conditions (happy or sad encoding context, upright or inverted test orientation). False alarm rates were determined separately for upright and inverted new faces incorrectly endorsed as old. Normalized hit and false alarm rates were used to calculate $d^{\prime}$ scores for each condition and participant. In order to correct for extreme hit and false alarm values (i.e., 1 or 0 ), we computed $d^{\prime}$ using the correction technique described by Snodgrass and Corwin (1988).

\section{Results and Discussion}

Recognition accuracy. Emotional context during learning systematically influenced the recognition results. As is shown in Figure 2A, HEC faces were remembered better than SEC faces when the test faces were upright. Conversely, SEC faces were remembered better than HEC faces when the test faces were inverted.

This outcome was confirmed with a two-way ANOVA (happy/sad encoding context $\times$ upright/inverted test orientation) on $d^{\prime}$ scores, with a significant interaction of context and orientation $[F(1,23)=8.25, p<.01]$. Further tests indicated a memory advantage for HEC relative to SEC upright faces $[t(23)=1.86, p<.08]$ and for $\mathrm{SEC}$ relative to HEC inverted faces $[t(23)=2.00, p<$ .06], but both comparisons only approached statistical significance. In addition, the main effect of orientation $[F(1,23)=12.73, p<.003]$ confirmed the typical memory advantage for upright faces $\left(d^{\prime}=0.94, S E=.11\right)$ relative to inverted faces $\left(d^{\prime}=0.38, S E=.11\right)$, and there was no overall effect of encoding context $(p>.7)$.

We also examined recognition accuracy for each condition separately (Figure 2B). Hit rates were higher for upright HEC faces than for upright SEC faces, whereas hit rates were higher for inverted SEC faces than for inverted HEC faces. A two-way ANOVA confirmed this interactive influence of emotional context as a function of face orientation $[F(1,23)=7.07, p<.02]$. There was also a main effect of orientation on hit rate $[F(1,23)=4.41, p<.05]$, reflecting the classic face-inversion effect. Further tests for hit rate differences between upright HEC and SEC faces $[t(23)=1.67, p<.11]$ and between inverted SEC and HEC faces $[t(23)=1.97, p<.07]$ failed to reach statistical significance. Finally, we compared false alarm rates for upright and inverted faces and observed a higher false alarm rate for inverted faces than for upright faces $[t(23)=2.48, p<.03]$.

The crossover interaction of emotional encoding context and stimulus orientation at test is consistent with the notion of a processing shift at encoding between focusing on featural versus configural information. Prior studies have similarly demonstrated that mood can influence local versus global processing strategies with respect to hierarchical visual stimuli (Gasper \& Clore, 2002) and memory for other-race faces (Johnson \& Fredrickson, 2005). The results from Experiment 1 did not include mood assessments, so we do not know whether the context effects on memory arose because the vignettes influenced observers' moods, as in the aforementioned studies, or whether perceiving emotional context was sufficient. In any event, it is likely that the emotional vignettes modulated the participants' active exploration and encoding of facial information, such that attention was directed more toward individual features during the SEC encoding blocks and more toward the configuration of features during the HEC encoding blocks. Even though the faces appeared neutral in affect, it is possible that the emotional context associated with the faces led to a bias in the perception of
A

Recognition Sensitivity

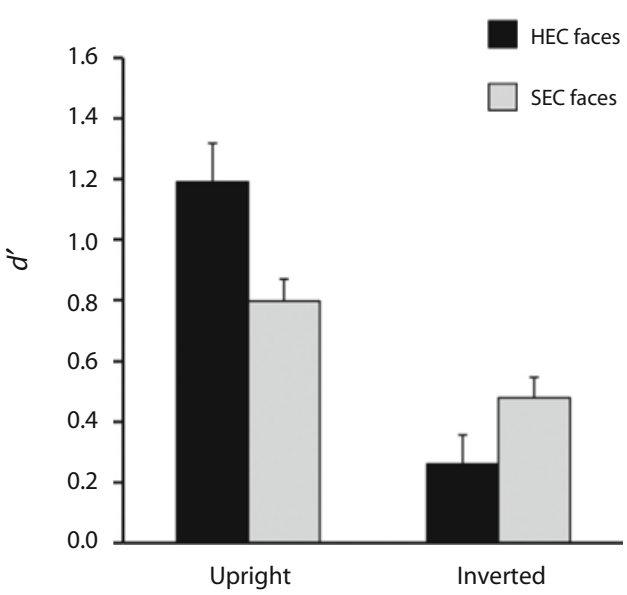

B

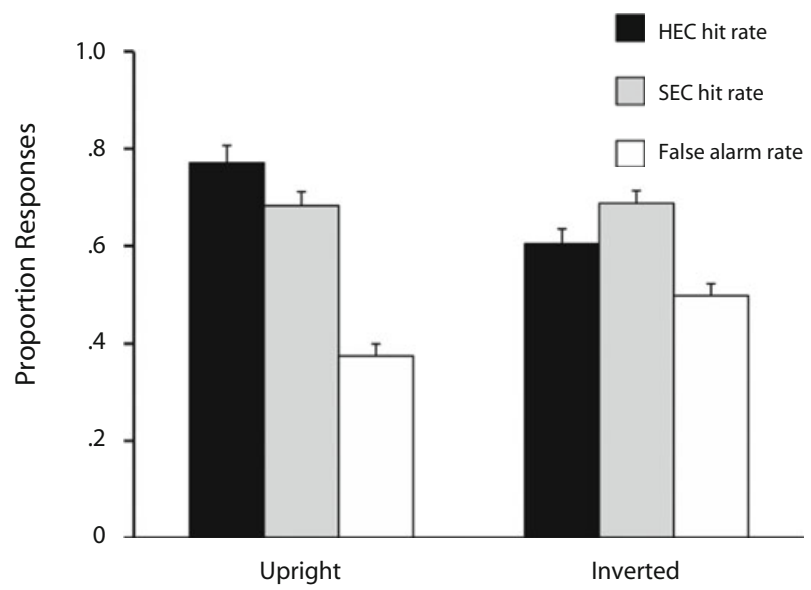

Figure 2. Recognition memory results from Experiment 1. (A) Discriminability scores $\left(d^{\prime}\right)$ for faces learned in a happy encoding context (HEC) or a sad encoding context (SEC), computed on the basis of results for upright and inverted faces presented during the test phase with corrected hit and false alarm rates. See the text for more details. (B) Raw hit rates for HEC and SEC faces along with false alarm rates for new faces. Error bars indicate 1 SEM. 
Table 1

Source-Memory Results

\begin{tabular}{|c|c|c|c|c|c|c|c|c|}
\hline & \multicolumn{4}{|c|}{ Upright } & \multicolumn{4}{|c|}{ Inverted } \\
\hline & \multicolumn{2}{|c|}{ Happy } & \multicolumn{2}{|c|}{ Sad } & \multicolumn{2}{|c|}{ Happy } & \multicolumn{2}{|c|}{ Sad } \\
\hline & $M$ & $S E$ & $M$ & $S E$ & $M$ & $S E$ & $M$ & $S E$ \\
\hline \multicolumn{9}{|c|}{ Experiment $1(N=24)$} \\
\hline Study trial hits & .50 & .04 & .63 & .04 & .46 & .04 & .56 & .04 \\
\hline All study trials & .48 & .03 & .57 & .03 & .53 & .04 & .51 & .03 \\
\hline Lures & .46 & .03 & .54 & .03 & .53 & .03 & .47 & .03 \\
\hline Source memory score & .04 & .06 & .09 & .05 & -.07 & .05 & .09 & .04 \\
\hline \multicolumn{9}{|c|}{ Experiment $2(N=20)$} \\
\hline Study trial hits & .60 & .05 & .59 & .06 & .59 & .04 & .56 & .04 \\
\hline All study trials & .54 & .05 & .62 & .04 & .53 & .04 & .58 & .04 \\
\hline Lures & .44 & .06 & .56 & .06 & .42 & .08 & .58 & .08 \\
\hline Source memory score & .17 & .05 & .03 & .06 & .16 & .05 & -.02 & .04 \\
\hline
\end{tabular}

Note-Study trial hits and all study trials show the proportion of faces in each specified condition for which the context type was classified correctly as happy or sad. Lures shows the proportion of happy and sad responses for upright and inverted faces, usually showing a bias for the sad response. Source memory scores for each condition were calculated as the proportion of hits correctly classified as happy or sad minus the proportion of lures classified as happy or sad for upright and inverted faces.

emotional information conveyed by the face. We explored these issues further in Experiment 2.

Source memory. Given that a source-memory advantage for happy facial expressions has been found previously (Shimamura et al., 2006), we predicted that HECs would be remembered more often than SECs. In order to interpret the accuracy of context decisions, however, it is necessary to take the possibility of a bias in context decisions into account. In other words, the guessing rate may not be $50 \%$ in this test if the participants had a bias to indicate a happy encoding context or a sad encoding context. We used responses to new faces to estimate guessing rates and computed a source-memory score for each condition by subtracting these estimates from corresponding context accuracy values for old faces.

Table 1 shows that source-memory accuracy was somewhat higher than $50 \%$ correct when considering only study trials in which the face was recognized as an old face (hits) or when considering all study trials. However, there was also a bias to select a sad context in response to lures. Mean source-memory scores, computed by subtracting lure results from hit results, were generally near chance levels (similar results were apparent if all study trials were used). No significant effects were found when these scores were analyzed in a two-way ANOVA (happy/ sad emotional context $\times$ upright/inverted test orientation). Furthermore, source memory scores averaged across conditions showed that context memory overall $(M=.04$, $S E=.03$; chance $=0)$ did not differ from chance $[t(23)=$ $1.44, p>.1]$. These findings suggest that source memory was very weak and that the emotional encoding context was not reliably available to the participants upon identification of the study faces. However, in light of possible floor effects in source-memory accuracy, definitive conclusions cannot be drawn in regard to the influence of emotional encoding context.

\section{EXPERIMENT 2}

In our next experiment, we aimed to provide a replication of the results observed in Experiment 1 and to address two additional questions. First, we sought to determine whether participants' moods were influenced by the valence of the emotional encoding blocks. A sad mood may result from reading the sad vignettes, and likewise, a happy mood may result from reading the happy vignettes. If so, mood might mediate the influence of encoding context on memory. Therefore, we altered the design by incorporating a self-report mood measure at the end of each study block.

Second, we investigated whether the participants' perception of study faces was altered as a function of the emotional encoding context. The vignettes may have modified the interpretation of emotional facial information, such that expressions perceived in HEC faces may have been shifted to appear more happy and those in SEC faces to appear more sad. Therefore, we included an affect-rating procedure in which the participants rated each face following the memory test.

\section{Method}

The materials and procedure were identical to those in Experiment 1 , except where otherwise noted.

Participants. Twenty Northwestern University undergraduates participated in the study for course credit (10 women; mean age $=$ 18.95 years, $S E=0.21$ )

Procedure. The participants were given the same instructions as were those in Experiment 1, except that they were also informed that they would periodically complete a mood-rating scale. At the end of each study block, the participants were instructed to rate their current mood on a scale of $1-9(1=$ very negative; $9=$ very positive $)$.

After the memory test phase, the participants were given instructions for the affect-rating procedure. In a random order, the entire set of 64 faces from the memory task was presented again. Each face was displayed upright and remained on the screen until the participants pressed a button to make a response. The participants were asked to rate the perceived valence of the face on a scale of 1-9 ( 1 = negative $; 9=$ positive $)$. The experiment ended after the affectrating procedure, and unlike in Experiment 1, the participants did not rate the affect of the vignettes.

\section{Results and Discussion}

Recognition accuracy. Recognition memory was systematically affected by emotional encoding context, as is shown in Figure 3A. Just as in Experiment 1, we observed an interaction of emotional encoding context and orientation on $d^{\prime}[F(1,19)=10.38, p<.005]$. Follow-up $t$ tests revealed a significant memory advantage for inverted SEC faces relative to inverted HEC faces $[t(19)=2.37$, $p<.03]$ and a nonsignificant trend of better memory for upright HEC faces than for upright SEC faces $[t(19)=$ $1.76, p<.1]$.

Although the critical interaction of emotion and orientation on recognition sensitivity was highly reliable in both Experiment 1 and Experiment 2, follow-up tests for effects of emotional context restricted to either upright or inverted faces often failed to reach significance. Given that strong trends were apparent in the expected directions, it is plausible that the number of participants 


\section{A Recognition Sensitivity}

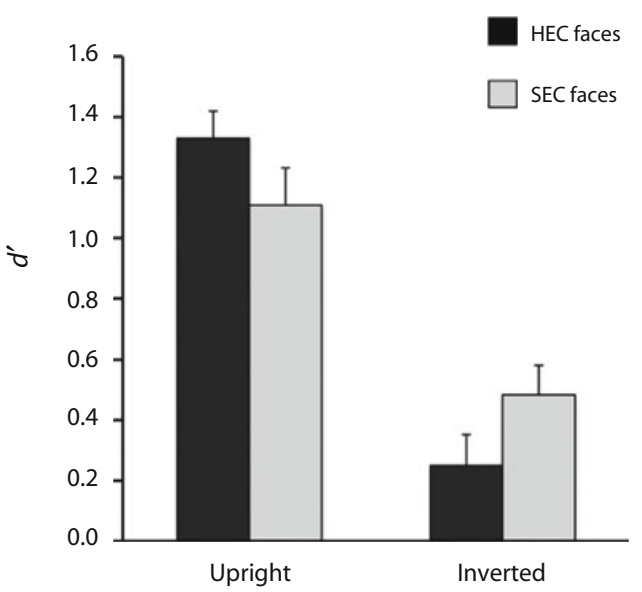

B

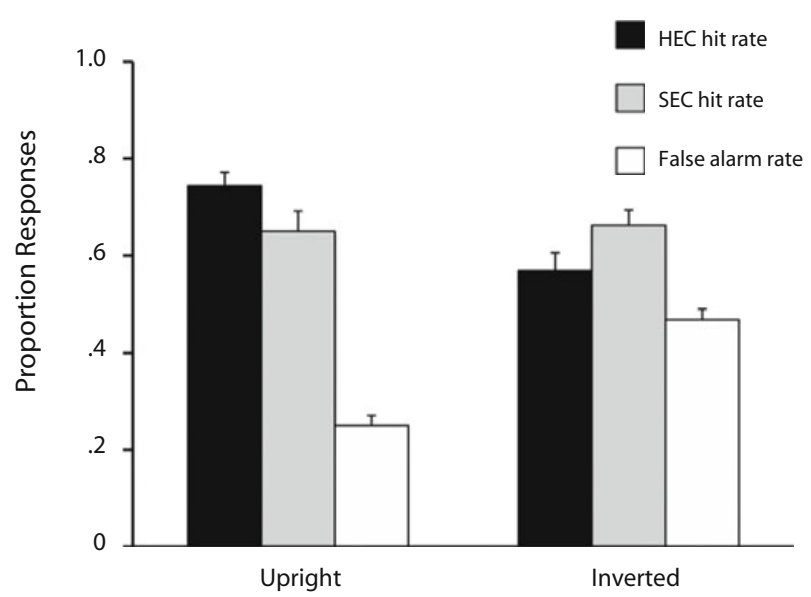

Figure 3. Recognition memory results from Experiment 2. (A) Discriminability scores ( $\left.d^{\prime}\right)$ for faces learned in a happy encoding context (HEC) or a sad encoding context (SEC), computed on the basis of results for upright and inverted faces presented during the test phase with corrected hit and false alarm rates. See the text for more details. (B) Raw hit rates for HEC and SEC faces along with false alarm rates for new faces. Error bars indicate 1 SEM.

in each experiment did not provide sufficient statistical power. An additional analysis was thus conducted using $d^{\prime}$ scores combined from the two experiments $(N=44)$. Differences in recognition sensitivity as a function of encoding context were substantiated for both upright faces $[t(43)=2.56, p<.02]$ and inverted faces $[t(43)=3.07$, $p<.005]$. Overall, upright HEC faces were remembered better than upright SEC faces $\left(d^{\prime}=1.19\right.$ and $d^{\prime}=0.94$, respectively), whereas inverted SEC faces were remembered better than inverted HEC faces $\left(d^{\prime}=0.48\right.$ and $d^{\prime}=$ 0.26 , respectively).

We also observed a main effect of orientation in Experiment $2[F(1,19)=24.52, p<.001]$; recognition accuracy for inverted faces $\left(d^{\prime}=0.34, S E=.12\right)$ was very poor relative to that for upright faces $\left(d^{\prime}=1.22, S E=.14\right)$. In keeping with this evidence of a face-inversion effect, inverted faces elicited more false alarms than did upright faces $[t(19)=5.22, p<.001]$. The main effect of encoding context was not significant $(p>9)$.

A hit rate analysis also revealed a significant interaction of emotional encoding context and orientation $[F(1,19)=$ $14.49, p<.002$ ], as is shown in Figure 3B. These results paralleled the $d^{\prime}$ results. Corresponding differences in hit rate were found between upright HEC and SEC faces $[t(19)=2.16, p<.05]$ and between inverted SEC and HEC faces $[t(19)=2.38, p<.03]$.

Mood ratings. Mood was significantly affected by emotional encoding context $[t(19)=5.45, p<.001]$. Following HEC blocks, the participants reported a more positive $\operatorname{mood}(M=5.53, S E=0.21)$ than after SEC blocks $(M=4.00, S E=0.28)$. This difference in reported mood raises the possibility that mood contributed to the crossover memory effect. To examine this possibility, we conducted a correlational analysis to determine whether the mood difference between HEC and SEC blocks was associated with the magnitude of the HEC-SEC memory effect for either upright or inverted faces. However, the correlational analysis did not reveal a significant relationship between mood differences reported following HEC relative to SEC blocks and the interactive memory effects observed for upright and inverted faces $(r=.12, p>.6$, and $r=-.08, p>.7$, respectively). Despite these nonsignificant correlations, it is possible that mood did play a role in the observed memory effects and that the correlational analysis was not powerful enough to reveal the relationship. Nonetheless, these results show that mood did indeed shift depending upon the encoding context, and thus, we cannot rule out the possibility that interactive memory effects are dependent on mood.

Facial affect ratings. At the time of encoding, emotional vignettes may have caused the participants to interpret emotional expressions of the nominally neutral faces as consistent with vignette valence. However, we elected not to acquire facial affect ratings during the study phase in order to avoid introducing an extra factor that might alter facial encoding. We instead acquired facial affect ratings after the test phase. The results failed to show any reliable influence of emotional encoding context on the participants' perceived affective ratings of the HEC, SEC, and lure test faces $[F(2,38)=1.46, p>.2]$. The ratings were similar for HEC faces $(M=4.71, S E=0.16)$, SEC faces $(M=4.54, S E=0.10)$, and lures $(M=4.63, S E=$ 0.11 ). We thus have no evidence to support the notion that the participants' perception of the emotional valence conveyed by the faces contributed to the observed recognition memory effects. We cannot rule out an influence on expression perception at the time of encoding that faded by the end of the experiment.

Source memory. We computed source memory scores using the results from recognition hits and from lures, as 
in Experiment 1. As is shown in Table 1, source memory scores were significantly greater than chance performance $[t(19)=2.98, p<.01]$. Furthermore, HECs $(M=.17$, $S E=.05)$ were remembered better than SECs $(M=.01$, $S E=.04)$, as was indicated by a main effect of emotional context in a $2 \times 2$ (emotional context $\times$ orientation) ANOVA $[F(1,19)=5.96, p<.03]$. Accuracy was nearly the same for study-trial hits, whereas there was a bias to select the sad-context response with new faces. Although these results are consistent with the source-memory advantage for happy facial expressions reported previously (Shimamura et al., 2006), the fact that they depend on an estimate of bias derived from new faces leads us to be cautious about interpreting these results, given that responses to new faces may have reflected either a source-memory bias or some evaluation of facial affect in these nominally neutral faces.

\section{GENERAL DISCUSSION}

Recognition memory can be influenced by the presence of emotional information and by the mood of the observer, as was reviewed above. In our experiments, the to-beremembered objects were ostensibly neutral-unfamiliar faces with neutral emotional expressions. Whereas these faces had minimal emotional content, emotional factors were manipulated independently using a context sentence that the participants read prior to encoding each face. The same facial images appeared again during the memory test. As was predicted, the results from both Experiment 1 and Experiment 2 showed systematic effects of encoding context on memory that varied according to whether faces were upright or inverted at test. Our interpretation is twofold: (1) The happy encoding context provoked better encoding of configural facial information than the sad encoding context, yielding better memory when recognition was tested with upright faces, and (2) the sad encoding context resulted in better encoding of individual facial components than the happy encoding context, resulting in better memory when recognition was tested with inverted faces.

Our experimental analyses were focused on the contrasts between the happy and the sad contexts at encoding. We did not include a neutral context at encoding, so further experimentation is needed to determine whether only one type of context was effective or whether both contexts were effective in altering face encoding. Nonetheless, we can conclude with certainty that the happy and sad contexts had differential effects at encoding and that these effects yielded differential memory performance at test as a function of face orientation. Moreover, these conclusions fit well with predictions derived from considerations of how emotion can systematically influence attention to global versus local stimulus information (e.g., Fredrickson \& Branigan, 2005; Gasper \& Clore, 2002).

These crossover memory effects provide novel evidence that memory can be modulated by emotions activated in the encoding context independent of emotional cues inherent in the stimuli, as in happy and sad facial expressions or positive and negative emotional scenes. When memory is examined using emotional facial expressions, for example, it is difficult to disentangle direct effects of explicit emotional cues and indirect effects of the emotions engendered by those cues. Because the emotional information was paired with neutral faces in our experiments, we can infer that face encoding was altered because of the concurrent emotional information that the participants processed and not because of facial configurations that coincide with certain expressions. On the other hand, it is unclear whether emotional contexts influenced memory by causing the participants to perceive the neutral faces as displaying corresponding emotional expressions.

Another factor that may have contributed to the observed memory effects is mood. Many studies have shown that depression and anxiety can influence how individuals process facial information (Jermann, van der Linden, \& D’Argembeau, 2008; Ridout et al., 2003; Yoon \& Zinbarg, 2008). Indeed, the self-reported mood of our participants was modulated in accordance with the vignettes that they read, in that more sadness was evident after sad-context blocks than after happy-context blocks; however, differential mood ratings did not correlate with either the inverted or the upright emotional context memory effects. Although individual differences in mood ratings were not obviously related to the observed memory effects, we cannot dissociate mood from the emotional encoding context and therefore cannot rule out the possibility that mood changes were operative in achieving the crossover pattern of memory effects reported here.

Even though the emotional memory effects described above are intriguing, this study has some limitations that could be addressed in future research. First of all, we do not know whether individual vignettes were powerful enough to influence encoding in isolation. Our design used blocks of eight vignettes of the same valence in order to take advantage of a cumulative emotional effect. Second, we do not know whether the particular circumstances of memory testing, such as the inclusion of a source-memory question, were essential for observing these effects. Third, as was mentioned above, we did not determine whether a happy context boosted holistic processing relative to a neutral context or whether a sad context impaired holistic processing relative to a neutral context. We also do not know whether other emotions might operate similarly. Finally, we did not map out the effects of retention delay. We used one delay, which was relatively short (about $11 \mathrm{~min}$, on average), so it remains possible that the memory advantages observed on the basis of encoding context would take a different form if a different retention delay were used.

In summary, the results from the present study underscore the importance of emotion and its influence on attentional focus for subsequent memory. Emotion can systematically bias facial encoding toward holistic or analytic facial information. Happiness might generally be superior for face memory relative to sadness, because we so seldom need to recognize inverted faces, but this benefit might reflect an emphasis on configural face processing at the expense of encoding isolated facial features. Given the robust influence of emotional encoding context on subsequent 
memory for faces, it may be useful to explore whether similar effects can be observed with other stimuli, such as neutral scenes, vocabulary words, or factual knowledge. Emotional encoding context, either arising from the to-beremembered information directly or arising independently, must be given suitable consideration in order to fully characterize the influence of emotion on memory.

\section{AUTHOR NOTE}

This work was supported by NSF Grants BCS-0518880 and BCS0818912 to K.A.P. and NSF Grants BCS-0720312 and BCS-0722326 to J.Y.C. The authors thank Joel Voss for programming assistance, Tim Sweeny for providing facial stimuli, and Jason Scimeca for assistance with stimulus preparation. We also extend our gratitude to Art Shimamura and Galit Yovel for providing insightful feedback on an earlier version of the manuscript. Correspondence concerning this article should be sent to D. J. Bridge, Department of Psychology, Northwestern University, Cresap Hall, 2029 Sheridan Rd., Evanston, IL 60208 (e-mail: donnajb@u.northwestern.edu).

\section{REFERENCES}

Adolphs, R., Gosselin, F., Buchanan, T. W., Tranel, D., Schyns, P., \& DAMASIO, A. R. (2005). A mechanism for impaired fear recognition after amygdala damage. Nature, 433, 68-72.

BARRETT, L. F., LiNDQUist, K. A., \& GENDRON, M. (2007). Language as context for the perception of emotion. Trends in Cognitive Sciences, 11, 327-332.

Canli, T., Zhao, Z., Brewer, J., Gabrieli, J. D., \& Cahill, L. (2000). Event-related activation in the human amygdala associates with later memory for individual emotional experience. Journal of Neuroscience, 20, 99-103.

D'Argembeau, A., \& van der Linden, M. (2007). Facial expressions of emotion influence memory for facial identity in an automatic way. Emotion, 7, 507-515.

D'Argembeau, A., van der Linden, M., Comblain, C., \& Etienne, A.-M. (2003). The effects of happy and angry expressions on identity and expression memory for unfamiliar faces. Cognition \& Emotion, 17, 609-622.

DiAmond, R., \& CAREY, S. (1986). Why faces are and are not special: An effect of expertise. Journal of Experimental Psychology: General, 115, 107-117.

Fredrickson, B. L., \& Branigan, C. (2005). Positive emotions broaden the scope of attention and thought-action repertoires. Cognition \& Emotion, 19, 313-332.

Gable, P. A., \& Harmon-Jones, E. (2008). Approach-motivated positive affect reduces breadth of attention. Psychological Science, 19, 476-482.

Gasper, K., \& Clore, G. L. (2002). Attending to the big picture: Mood and global versus local processing of visual information. Psychological Science, 13, 34-40.

Golby, A. J., Gabrieli, J. D., Chiao, J. Y., \& Eberhardt, J. L. (2001). Differential responses in the fusiform region to same-race and otherrace faces. Nature Neuroscience, 4, 845-850.

Jermann, F., van der Linden, M., \& D'Argembeau, A. (2008). Identity recognition and happy and sad facial expression recall: Influence of depressive symptoms. Memory, 16, 364-373.

Johnson, K. J., \& Fredrickson, B. L. (2005). "We all look the same to me": Positive emotions eliminate the own-race bias in face recognition. Psychological Science, 16, 875-881.

Kensinger, E. A., Garoff-Eaton, R. J., \& Schacter, D. L. (2007a). Effects of emotion on memory specificity in young and older adults. Journals of Gerontology: Psychological Sciences, 62B, 208-215.

Kensinger, E. A., Garoff-Eaton, R. J., \& Schacter, D. L. (2007b). Effects of emotion on memory specificity: Memory trade-offs elicited by negative visually arousing stimuli. Journal of Memory \& Language, 56, 575-591.

Kim, H., Somerville, L. H., Johnstone, T., Polis, S., Alexander, A. L., Shin, L. M., \& WhaLen, P. J. (2004). Contextual modulation of amygdala responsivity to surprised faces. Journal of Cognitive Neuroscience, 16, 1730-1745.

Lundqvist, D., Flykt, A., \& Ohman, A. (1998). The Karolinska directed emotional faces [CD-ROM]. Stockholm: Karolinska Institute, Psychology section, Department of Clinical Neuroscience.

Marian, D. E., \& Shimamura, A. P. (2004). Effects of emotional facial expressions on memory for faces and associate expressions. Paper presented at the 10th Annual Meeting of the Cognitive Science Association for Interdisciplinary Learning, Hood River, OR.

Maurer, D., Le Grand, R. L., \& Mondloch, C. J. (2002). The many faces of configural processing. Trends in Cognitive Sciences, $\mathbf{6}$, 255-260.

NAvON, D. (1977). Forest before trees: The precedence of global features in visual perception. Cognitive Psychology, 9, 353-383.

OCHSNER, K. N. (2000). Are affective events richly recollected or simply familiar? The experience and process of recognizing feelings past. Journal of Experimental Psychology: General, 129, 242-261.

Payne, J. D., Stickgold, R., Swanberg, K., \& Kensinger, E. A. (2008). Sleep preferentially enhances memory for emotional components of scenes. Psychological Science, 19, 781-788.

Phelps, E. A. (2004). Human emotion and memory: Interactions of the amygdala and hippocampal complex. Current Opinion in Neurobiology, 14, 198-202.

Ridout, N., Astell, A. J., Reid, I. C., Glen, T., \& O'Carroll, R. E. (2003). Memory bias for emotional facial expressions in major depression. Cognition \& Emotion, 17, 101-122.

Rowe, G., Hirsh, J. B., \& Anderson, A. K. (2007). Positive affect increases the breadth of attentional selection. Proceedings of the $\mathrm{Na}$ tional Academy of Sciences, 104, 383-388.

Shimamura, A. P., Ross, J. G., \& Bennett, H. D. (2006). Memory for facial expressions: The power of a smile. Psychonomic Bulletin \& Review, 13, 217-222.

Snodgrass, J. G., \& Corwin, J. (1988). Pragmatics of measuring recognition memory: Applications to dementia and amnesia. Journal of Experimental Psychology: General, 117, 34-50.

Sweeny, T. D., Grabowecky, M., Suzuki, S., \& Paller, K. A. (2009). Long-lasting effects of subliminal affective priming from facial expressions. Consciousness \& Cognition, 18, 929-938.

TANAKA, J. W., \& FARAH, M. J. (1993). Parts and wholes in face recognition. Quarterly Journal of Experimental Psychology, 46A, 225-245.

Tyler, C. W., \& Chen, C.-C. (2006). Spatial summation of face information. Journal of Vision, 6 (10, Art. 11), 1117-1125. doi:10.1167/ 6.10 .11

Valentine, T. (1988). Upside-down faces: A review of the effect of inversion upon face recognition. British Journal of Psychology, 79, 471-491.

Yoon, K. L., \& ZinBarG, R. E. (2008). Interpreting neutral faces as threatening is a default mode for socially anxious individuals. Journal of Abnormal Psychology, 117, 680-685. 


\section{APPENDIX}

\section{Happy Vignettes}

1. Lindsey received freshly baked cookies in the mail today from her mom.

2. Jennifer's friends threw her a surprise birthday party.

3. Alicia received an $\mathrm{A}+$ on her final exam in a very challenging class.

4. Tara won first place in his event at the swimming meet yesterday.

5. Amelia is anticipating a visit from her best friend this weekend.

6. Brianna was excited to find out that she was accepted to her first choice university.

7. Caroline won the lottery and was able to get her family out of debt.

8. Mary was awarded a prestigious scholarship to study abroad at a university.

9. Jordan's favorite football team won the Super Bowl this year.

10. Adam set a new school record for the number of points he scored this basketball season.

11. After only one month at his new job, James was promoted.

12. Geordie is a photographer and will have his first gallery show this spring in NYC.

13. Jack is going on vacation to Hawaii for one month.

14. Tyler asked a girl he had a crush on out on a date and she said "yes."

15. Stone wants to be a director. He made a short film in college and it won many awards.

16. Marc and his wife adopted their first child last fall.

\section{Sad Vignettes}

1. Elaine's five-year-old son went missing a year ago.

2. Jessica miscarried her first child.

3. Shelly was recently diagnosed with breast cancer.

4. Sara and her family are living in a shelter because their house burned down.

5. Casey lost a precious heirloom given to her by her mother before she died.

6. Rachel's brother is in the hospital because he overdosed on heroin.

7. Martha's grandmother has Alzheimer's disease.

8. Rebecca trained for months leading up to the triathlon but broke her leg a week before the competition.

9. Tom's best friend unexpectedly died a few days ago.

10. Richard was in a car accident and is now paralyzed from the waist down.

11. As a child, Anthony was physically abused by his stepfather.

12. Steven lost his entire thesis project when his computer crashed during a thunderstorm.

13. All of Max's friends forgot his birthday this year.

14. Christopher was forced to drop out of school because he could no longer afford to pay tuition.

15. Mitch was upset to find out that his parents are getting a divorce.

16. George's parents are unable to accept his lifestyle choices.

(Manuscript received April 20, 2009;

revision accepted for publication August 31, 2009.) 